\title{
Using the Physics of Notations Theory to Evaluate the Visual Notation of SEAM
}

\author{
George Popescu \\ Systemic Modeling Laboratory (LAMS) \\ École Polytechnique Fédérale de Lausanne (EPFL) \\ CH-1015 Lausanne, Switzerland \\ george.popescu@epfl.ch
}

\author{
Alain Wegmann \\ Systemic Modeling Laboratory (LAMS) \\ École Polytechnique Fédérale de Lausanne (EPFL) \\ CH-1015 Lausanne, Switzerland \\ alain.wegmann@epfl.ch
}

\begin{abstract}
Modeling languages are used to make models that are commonly used in communicating about real-life situations such as the modeling of business and IT requirements in organizations. A way to evaluate how effective the modeling languages are for communicating their intended messages is to use the set of nine principles defined in the Physics of Notations Theory (PoNT, Moody). PoNT helps designers evaluate the notation of modeling languages and provides guidelines for improving it. We apply this theory to evaluate the visual notation of a systemic method, the Systemic Enterprise Architecture Methodology (SEAM) that is designed to model business and IT requirements. In order to make the SEAM notation more cognitively effective, we identify some notation limitations and provide specific recommendations for improvement for each of the nine PoNT principles.
\end{abstract}

Keywords-notation, Physics of Notations Theory, business and IT alignment, business design, IT design, SEAM.

\section{INTRODUCTION}

Visual notations are used in all areas of business and IT practice, from strategic planning to the design of IT systems. They play a critical role in communicating with internal and external stakeholders such as the design team and customers. The main purpose of a model is to support the construction of a common understanding between all stakeholders. Moody [7] mentions that the desirable goals for a notation are to maximize precision, expressiveness and parsimony. These attributes should enhance the communication among business and/or IT stakeholders. The field of visual notation evaluation includes several visual notation evaluation frameworks such as: Cognitive dimensions of notations [3] SEQUAL framework [5], Seven process modeling guidelines [6] and Physics of Notations Theory (PoNT) [7]. We chose PoNT as it is the state of the art SE and RE notation evaluation frameworks widely used with other notations. PoNT is applied to evaluate and improve the visual notation of some of the leading SE and RE notations such as i* (goal modeling language [8]), ArchiMate (modeling language for enterprise architecture [7]), UML (international standard for modeling software systems [9]), Use Case Maps (user requirements notation [1]), Business Decision Modeling (decision modeling notation [13]) and BPMN (international standard for business process modeling [2]). Moody [7] mentions the lack of principles for designing visual notations, as designers rely on instinct, imitation and tradition.
As a solution, Moody proposes to involve business and IT representatives in the notation design process.

SEAM is used for the analysis and the design of business and IT systems involving various stakeholders (business and IT representatives, clients, etc.). A SEAM model shows a number of different model views, e.g., the business view showing business concepts such as companies, customers, and suppliers, and the IT view showing IT concepts such as infrastructure, applications, and servers. The model shows the services provided by the different entities participating in a process. This can be applied to business as well as to IT services or processes. SEAM designers model both the business and the IT, therefore they analyze and design the alignment between the business and the IT systems.

The research objective of this paper is to assess the SEAM notation using PoNT - an evaluation framework that helps understand how people perceive models. We evaluate the SEAM visual notation to determine its overall fit to the human cognitive system through the lenses of PoNT. The guidelines provided by each of the nine PoNT principles are useful for improving the SEAM visual notation. We discuss the general implication of evaluating modeling languages for business and IT. Thereby we promote the use of PoNT for other visual modeling notations, developed in the academia or industry. The critique of the SEAM notation is useful for EA modelers and SEAM designers, given the large number of projects using SEAM (academia and industry). It shows how this type of analysis can be used to evaluate other methods similarly.

Section II provides an overview of SEAM. In Section III, we give a general description of the PoNT visual notation framework. In Section IV, we evaluate the SEAM notation with the nine PoNT principles. In Section V, we include related work citing evaluations of other modeling methods with PoNT. We end the paper with conclusions and planned future work.

\section{SEAM OVERVIEW}

\section{A. SEAM Method and Theory}

SEAM is a systemic method [12]. It is grounded in systemic principles that are applied in business and in IT in the same way. Even if the same systemic principles are used, the modeling language looks different in business and in IT. SEAM is mainly applied for workshops to develop a shared 
understanding between business and IT stakeholders. Modelers represent service systems (system providing a service). The service is implemented by a process that combines services of lower-level service systems (Fig. 1). One of the key aspects of modeling with SEAM is the understanding of boundaries and the roles and responsibilities of the different entities. In SEAM, we develop concrete models that relate to the senses, emotions and real-life experiences of the people looking at the models. The goal in a SEAM workshop is to shape a coherent and convincing storyline for all the participants. The storyline illustrates the strategy developed and can be documented to promote the business / IT strategy.

In Fig. 1, we show the three main concepts of SEAM defined as some examples of semantic constructs: service, process and service system. The interpretation of the model is described as: "in a segment, the organization provides a service implemented by a process to the client". We represent the three service systems: segment, organization and client. We add also two behavioral elements: oval for service and hexagon for process. The service systems can have different shapes depending on the nature of what is represented. The arrow boxes represent organizations (companies, departments), and market structures (markets, segments). The stickman represents a human actor. In the notation, we use graphical elements inspired from existing notation (e.g., block arrow from Porter's theories [10], stickman from UML [9]). The composition rules are shown with straight lines, from service to process (meaning that the company provides a service that is implemented by the process) and from process to client (the client participates in the process). The boundary around objects defines the context in which they are represented (the company and the client are within a segment). In SEAM we consider this second kind of composition rules showing service systems as wholes (company and client) inside service systems as composites (segment) as important as the association between elements.

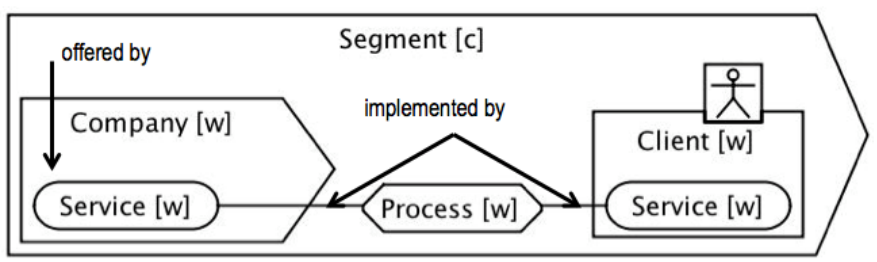

Fig. 1. Simple Example of SEAM visual notation and semantics.

SEAM is based on the RM-ODP standard that defines abstraction and refinement [4]. Abstraction is "the process of suppressing irrelevant detail to establish a simplified model, or the result of that process" [4]. Concepts are derived from the usage and classification of "real" or "concrete" concepts. Refinement is "the process of transforming one specification into a more detailed one" [4]. SEAM designers refer to the fact that the whole is refined into the composite. The whole view shows services visible from outside the boundary. The composite view shows the sub-entities and the services they provide while participating in processes.

In Fig. 2 the organizational hierarchy shows that the Service System as a whole [w] (black-box) is refined into the Service System as a composite [c] (white-box) or, vice-versa, the Service System [c] is abstracted into the Service System [w] (organizational hierarchy). The functional hierarchy shows the functional refinement of a behavioral element (service / process) as a whole into a composite. On the first row, the service [w] is refined into a [c] containing Sub-Service 1 and Sub-Service $2[\mathrm{w}]$. Next, the process [w] is refined into a [c] containing three sub-processes $[\mathrm{w}]$, each connected to a corresponding sub-service offered by corresponding service systems. The process [w] from the second row implements the corresponding service [w] from the first row. Seen as [c], the service implementation process is split into three subprocesses, the first implements the sub-service A and the last two implement the sub-service B.

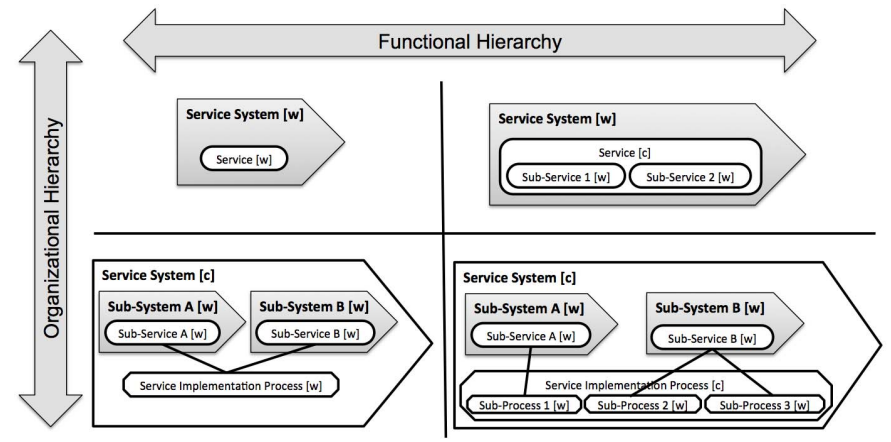

Fig. 2. Organisational and functional hierarchy example with SEAM.

\section{B. SEAM Models Notation}

The SEAM models are developed mostly in workshops by using Post-its. We also draw models to document the results; this is done often with hand drawings to keep the emotional contents of the workshops. For research purposes, we developed a modeling tool called SeamCAD. The illustrations of this paper are made using this tool. A web version of SEAM that helps entrepreneurs assess their business plan is [14].

The generic version of SEAM is called PureSEAM. PureSEAM is the common model describing a hierarchy of service systems. In SeamCAD, the hierarchy is built automatically for simple models. PureSEAM connects the three specialized views of SEAM, which can be considered as patterns applied on PureSEAM:

- Services and processes modeling (Behavior view).

- Motivations modeling (Goal-Belief view).

- Components, features, values mapping modeling (Supplier-Adopter Relationship model).

Even if these three versions have models, they can be understood as views of one common model. SEAM modelers analyze the motivations of the people, then they show how these motivations map to behavior and finally they interpret how this behavior helps create value for the customer. In this paper, we do not present how these different views are integrated. We consider them as independent models.

We evaluate the SEAM notation including Behavior, GoalBelief and Supplier-Adopter Relationship (SAR). In Table I we show the SEAM notation for: (1) service systems: human, business, IT, storage, facility and application (component in UML), (2) Behavior: localized action, localized property, 
action binding, undirected and identity relation, (3) GoalBelief: reduction, goal, belief, directed and identity relation, (4) SAR: reduction, supplier, adopter, (return) component, (return) feature, and (return) value. Service systems are represented separately because they are common for all three views.

TABLE I. SEAMCAD GRAPHICAL SYMBOLS

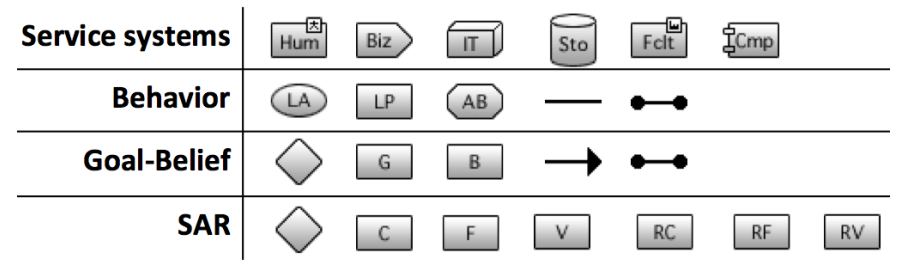

\section{1) Behavior View}

The SEAM Behavior view describes services of the business and IT organizations, and their corresponding implementation process. This is achieved through a hierarchy of systems and behavioral elements (Fig. 2).

In Fig. 3, the SEAM Behavior example includes different types of entities commonly represented in SEAM models such as technical infrastructure, external providers, and business and external users connected to IT and business processes. The model shows how IT provides a service to the business organization, which provides two services for two users. The Business $+[\mathrm{w}]$ and IT organizations $+[\mathrm{w}]$ include provided services and the [c] includes the details of the process that implements the services. The IT organisation+ is the IT organisation together with external providers. The Business organisation + is the business organisation, a business user and a specialist. Thus, from inside to outside, the IT organisation+ provides an IT service that is implemented by an IT process. The business process connects the services offered by the three entities seen as wholes: IT organisation+, business user and business specialist. At the next level, the Business organisation + provides a business service implemented by the business process. The process for internal / external user connects the business organisation and the two users.

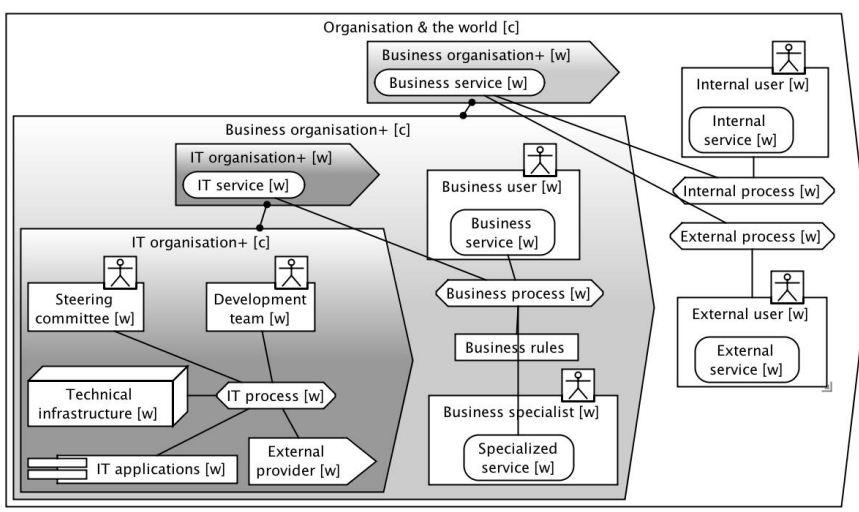

Fig. 3. Behavior example with SEAM.

\section{2) Goal-Belief View}

The SEAM Goal-Belief view describes the motivations of the business and of the IT stakeholders. Designers model the beliefs of the actors and the reality aspects that drive them to perform actions and achieve goals. The Goal-Belief view is based on Vicker's Appreciative System [11] and includes three types of judgment: readiness to see (reality judgment), readiness to value (value judgment) and readiness to act (action judgment).

In Fig. 4, the SEAM Goal-Belief example shows the connections between the values, realities and actions of two persons. A student (Marcel) and a company (represented by the HR recruiter Sam) participate at a university job fair. Marcel's main goal is to get a job in a good company and Sam's is to recruit a talented student. Marcel's beliefs can be related to the environment (realities, e.g., Sam's company is recruiting students with certain skills) or his inner values (e.g., Sam's company is a good employer and Marcel has some skills / knowledge that the company is looking for). By improving his skills, Marcel influences the belief (reality) of Sam who notices that the student has attained the required skill and thus decides to employ him. The associations for the same goal-belief model include stereotypes (belief and goal), which are alternatives to different graphical symbols. It allows keeping the link with the generic concept (property) from PureSEAM. Here we have a property goal, which is a specific use of a property in this version of SEAM, connecting the different versions of SEAM.

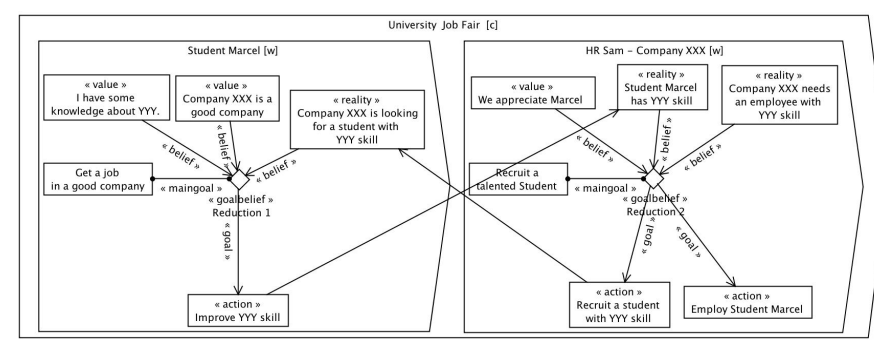

Fig. 4. Goal-Belief example with SEAM.

\section{3) Supplier-Adopter Relationship View}

The SEAM SAR view describes the relationship between a supplier and an adopter by using components, features and values that are mapped together in a dual hierarchy and a matrix view. The company is represented both as a composite (showing the components) and as a whole (showing the features). Adopters are represented to the right with the respective values they perceive.

In Fig. 5a, a SEAM Supplier-Adopter Relationship example shows the supplier (company) providing certain service components (IT service, data maintenance and incident management), which map to service features ( $24 \times 7$ support, membership and premium advantages), which are perceived as values by the business partner (more customers and databases) and the individual partner (data management and email support). The supplier is represented in the company value network context as a composite, containing the IT department+ as a whole. In the matrix view (Fig. 5b) the relation between components, features and values is established with check boxes. The positive and negative correlation is measured with three levels of green $(+)$ and red (-) symbols. When we compare the two representations (Fig. 5a and 5b), we note the consistency of meaning when passing from left to right, i.e., from service components (left) offered by the supplier, to the 
features (middle) and then to the values (right) perceived by the two types of partners.

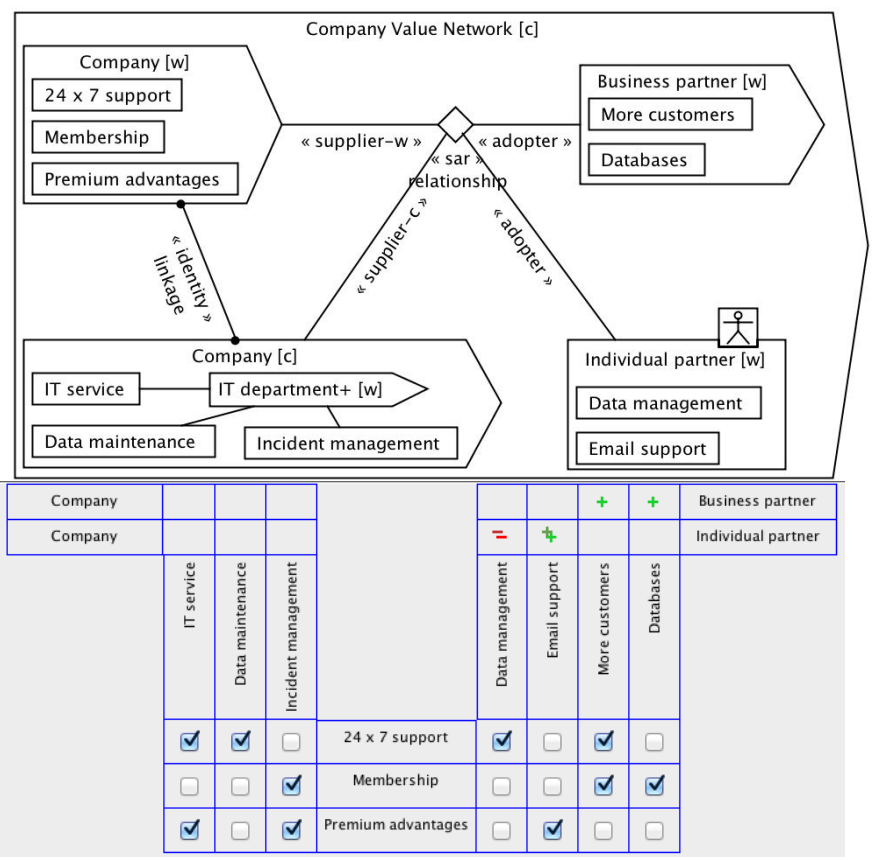

Fig. 5. Supplier-Adopter Relationship (SAR) example with SEAM (a) and the alternative matrix view of the same diagram as a dual coding concept (b).

\section{PHYSICS OF NOTATIONS THEORY}

PoNT is a framework for evaluating, comparing, improving and designing visual notations. The anatomy of a visual notation consists of (1) visual vocabulary: graphical symbols, (2) visual grammar: composition rules and (3) visual semantics: definitions of the meaning of each graphical symbol. The visual vocabulary (1) and the visual grammar (2) form the visual syntax. Graphical symbols are used to show semantic constructs, typically defined with a meta-model [7].

PoNT provides nine principles of notation evaluation that act as guidelines for designing a cognitively effective visual notation optimized for human communication and problem solving: semiotic clarity, perceptual discriminability, semantic transparency, complexity management, cognitive integration, visual expressiveness, dual coding, graphic economy, and cognitive fit. The nine principles were synthesized from theory and empirical evidence from a wide range of fields and rest on an explicit theory of how visual notations communicate [7].

\section{SEAM Notation EVALUATION USING PoNT}

Hereafter, we analyze the SEAM visual notation with all PoNT principles. For each, we begin with a synthetic definition and discuss how it applies to the SEAM notation.

\section{A. Semiotic Clarity}

Definition: "There should be a 1:1 correspondence between semantic constructs and graphical symbols" [7].
Moody mentions four possible ways to not achieve a 1:1 correspondence: redundancy (multiple graphical symbols are used for the same semantic construct), overload (multiple semantic constructs are used for the same graphical symbol), excess (a graphical symbol lacks semantic constructs) and deficit (a semantic construct lacks graphical symbols).

In applying this principle to the SEAM visual notation, we identified the issue of the level of semantic constructs (similar to [13]). As SEAM is used to represent a hierarchy of systems, semantic constructs are hierarchical (Fig. 2). In SEAM, behavior and state related elements are generic (meta-model) and have a generic representation. The service systems (working objects) have a generic meta-model but a specific icon (yet with similarities). The service system symbols represent the nature of the objects that are represented. This does not imply the need to change the symbol when delving into the hierarchy. Thus, there is a 1:1 correspondence for behavioral elements: oval for service and hexagon for process (Fig. 1). They all have a different graphical symbol and are designed to allow for refinements (icons in icons). The service system has a 6:1 relationship (Fig. 6). They all correspond to a service system. This is not symbol redundancy because the six graphical symbols represent different kinds of entities in the reality (e.g., enterprise, committee and department for business). This helps us to make the model readable. The shapes have some commonalities, so we recognize that they are of the same kind in the model. Their differences, however, show the specific ontological commitment.

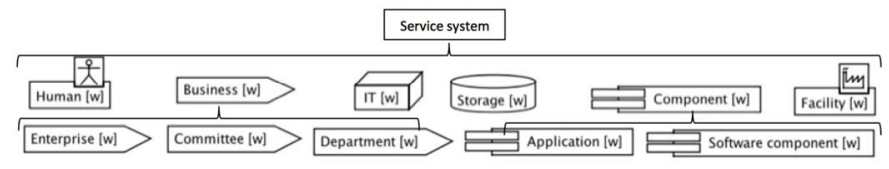

Fig. 6. Semantic constructs and graphical symbols at different hierarchical levels (top $=$ metamodel, middle $\&$ bottom $=$ upper $\&$ lower hierarchy $)$.

The SEAM notation uses a few types of relations: undirected between systems and processes, directed (link beliefs to the relevant goals in two Goal-Belief models, Fig. 4) and refinement (undirected linking whole to composite). Relations are also refined but the visual representation does not show this. A relation is a line, not a surface; so detailed working objects cannot be shown inside. Relations are also ambiguous because the same line connects Goal-Belief elements (in Fig. 4 the directed line connecting a reality or a value to the goal-belief reduction is the same as the line connecting the action of a system to the reality or value of another). The undirected line connecting the SAR components to features is the same as the line connecting features to values (Fig. 5a). The matrix view of the SAR (Fig 5b) complements the SAR model (the later is not supposed to show the details). The undirected line connecting the services to processes is the same as the one connecting services to properties in the Behavior view (Fig. 3). These are potential deficits of the notation. Providing different visual symbols for all types of relations is not a solution because the high number of possibilities is restricted by graphical economy. We use stereotypes instead. To sum up, we note that PoNT has difficulties in evaluating SEAM because constructs and symbols might not follow a 1:1 mapping in the hierarchy. 


\section{B. Perceptual Discriminability}

Definition: "Symbols should be clearly distinguishable from one another". This distinction can be achieved by increasing the visual distance between symbols, which is measured as the number of visual variables plus the size of differences [7].

The SEAM notation includes shapes similar to those of other RE and SE notations: $i^{*}$, UML, BPMN, etc. Service systems from the perceived reality are meant to be "special": drawn in 3D (IT infrastructure), with a distinctive icon at the top right of the graphical symbol in 2D (stickman), as block arrow (organizations). The services, processes and properties are always ovals, hexagons and rectangles.

Perceptual discriminability in SEAM is based on five variables: position (graphical symbols positioned to the top or to the right of the diagram traditionally show the company management / business, the supply chain goes from left to right), value (text labels), color (used mostly for grouping), orientation (horizontal for systems and any for links) and shape (dependent on the type of service system). The modeler can combine position and color to add additional unstructured information. This reflects the values of the modeler and is not currently specified in SEAM. In order to tell a story, SEAM uses layout features such as: grouping of similar elements, showing / hiding and coloring elements for extra information.

Suggestions for improvement include the use of the other two visual variables: texture (distinguish whole and composite) and size (elements can be as large as needed, show influence). Using SEAM modelers are flexible in defining the meaning associated with each visual variable (e.g., position similar elements together, use same color for services in the same diagram or for the organization and the services provided).

\section{Semantic Transparency}

Definition: "Notations should use graphical symbols whose appearance suggests meaning". The meaning (semantics) of a symbol should be clear from its appearance alone. Graphical symbols can be evaluated from +1 to -1 , or from semantic transparent to semantic perverse (opposite meaning) [7].

In SEAM, semantic transparent symbols can be used directly in workshops, e.g., stickman. Furthermore, participants create their own ad-hoc notation that allows them to focus on particular types of systems. Among the most common visual variables there are: position, color and size used to customize the story, also including grouping and stereotypes.

The SeamCAD notation includes simple objects. The oriented rectangle (business organizations), rectangle (properties, goals and beliefs), oval (services) and hexagon (processes) are all examples of poor semantic transparency.

As examples of types of associations between symbols and meaning [7] we mention the following:

- perceptual resemblance (icons): stickmen for people, plants for buildings and cylinders for databases.

- common logical properties: whole and composite views of service systems and behavioral elements (services and processes) represented with an extra label $[\mathrm{w} / \mathrm{c}]$.
- visual functional similarities: components, comments and orientation.

- metaphors: photos of real-life entities.

- culturally / learned associations: small add-ons, such as $+, \mathrm{x}, \sim$ and ! correspond to created, deleted, updated and problematic instances of graphical symbols.

Suggestions for improvement include the use of metaphors for graphical symbols (besides photos), the design of perceptual resembling icons for all working objects, the consistency of notation (different icon at the top right of each symbol) and the graphical distinction between wholes and composites (background texture, e.g., black for wholes and white for composites instead of the textual distinction: $[\mathrm{w} / \mathrm{c}])$.

\section{Complexity Management}

Definition: "Notations should include explicit mechanisms for dealing with complexity, such as modularization and hierarchy (abstraction)" [7].

SEAM is based on the service system as a whole and system as a composite approach (organizational hierarchy) and the service / process as a whole / composite approach (functional hierarchy). Designers use abstraction/refinement to represent elements of interest. The increased complexity modeled with a system as a composite that contains subsystems can be hidden by modeling the same system as a whole that shows the main services offered, without details.

Modularization ("dividing large diagrams into cognitively and perceptually manageable parts" [7]) can be achieved through highlighting / color-coding. It is aimed for classifying elements. For instance, SEAM designers can distinguish between different customer segments color-coding the types of services offered by the organization, services which are targeted to these specific segments.

\section{E. Cognitive Integration}

Definition: "Notations should integrate information between separate diagrams". Cognitive integration mechanisms refer to conceptual integration (summarization and visual momentum) and perceptual integration (signposting, orientation and navigation map) [7].

Perceptual integration mechanisms are not explicit in SEAM. This is because readers can build a story starting from any part of a SEAM model, for instance from the IT to the client or vice-versa in the Behavior view (Fig. 3), from the beliefs of the student to the goals of the HR employee, from the main goals of the two in the Goal-Belief view (Fig. 4), or from the supplier to the adopter or vice-versa in the SAR view (Fig. 5). Thus, navigation cues for signposting are not shown. Way finding contains four stages: orientation, route choice, route monitoring and destination recognition. It is implicitly expressed through the orientation and the navigation maps and through the whole/composite refinement/abstraction process (in Fig. 3 the IT organisation+ [w] is refined in the [c] and the Business organisation $+[\mathrm{w}]$ is refined in the $[\mathrm{c}])$. Models are either read from top to bottom (vertically), from right to left (horizontally), from business to IT, or from bottom to top / left 
to right. The model helps us understand how the IT provides IT services to the business who provides business services to the end customers. This shows the overall process in SEAM, starting from understanding business and IT requirements to designing the business and the IT systems. Thus, much emphasis in SEAM is put on understanding the surrounding service systems / environment. This allows modelers to narrow their analysis by navigating from the business environment to the specific IT applications (and code). The matrix alternative of SAR (Fig 5b) is a concise way to show relations and navigations between models. For instance, modelers first consider the service system / service / process as a whole, and then they model its details (composite). At each step of the modeling process with SEAM, they consider the organizational or functional refinement (separately or both in the same time).

Visual momentum is not explicitly captured in a SEAM diagram because modelers can concentrate on either the implementation of a service (which they call a process), the service itself, the resources needed for it, or the stakeholders involved. The "immediate family" principle applied to SEAM states that SEAM diagrams include directly related elements from other sub-diagrams; this is shown through the hierarchy.

\section{F. Visual Expressiveness}

Definition: "Notations should use the full range of the 7 visual variables: position, size, value, texture, color, orientation and shape" [7].

Similar to other SE and RE notations such as $i^{*}$, UML, ArchiMate, the SEAM notation uses less than the full range of the seven visual variables. It includes: value (labels for text), color (black is used by default but any colors are possible) and shape (boxes, oriented rectangles, diamonds, rectangles, circles, arrows, etc.). It either does not use or uses in an implicit manner the other variables: position (implicit wholes at the top and composites at the bottom), size (not used), texture (not used) and orientation (objects use only one orientation and connectors link objects at any angle). On the one hand, a "good" notation needs to use all visual variables and, on the other, the number of graphical symbols should be cognitively manageable (graphic economy principle). Furthermore, these variables need to be sparse for a generic diagram (fewer variables) and dense (more variables) for a domain specific one (as observed in [13]). In SEAM, the differentiation is realized as we delve into the hierarchy. Also, some variables influence the others, such as brightness influences color and texture influences color and brightness. Positioning (expressed through vertical and horizontal coordinates) is influenced by the presence of a grid ([13]). The orientation of the elements can have different values depending on shape: services drawn as ovals have only 2: larger on the $\mathrm{x}$ or on the $\mathrm{y}$-axis, processes drawn as hexagons have 3 : oriented at 0,30 or 60 degrees.

\section{G. Dual Coding}

Definition: "Notation should use text to complement (not replace) graphics". Textual annotations should be used alongside graphics [7].

In SEAM, textual annotations are used through short expressions or sentences for the service systems' names, goals, beliefs and services/processes. There are no graphical symbols without names. In addition, we use the same (partial) name of objects to group elements of the same type. As some graphical symbols represent certain categories, they do not need to include the name of the category in the label, e.g., the cube always represents a "technical infrastructure", the application a "software component", and the silo a "database".

Stereotypes in SEAM are marked as "<<type $>>$ ". In the Behavior view they are used for different types of properties. In the Goal-Belief view, they are useful for marking the maingoal, goals or beliefs (Fig. 3), or specific types of beliefs (reality, value, and action). In the SAR model stereotypes are used for the identity linkage, supplier and adopter.

SEAM is designed to tell stories. As a consequence, the text (names, actions, expressions) from the SEAM models contributes to shaping the story. Currently, there is no automatic tool support that allows passing from a formal model to a story, but from our experience this is feasible.

\section{H. Graphic Economy}

Definition: "The number of graphical symbols should be cognitively manageable". A large number of conventions increase complexity, thus reducing understanding [7].

Complexity is measured by the number of different conventions made in models. The larger semantic and graphic complexity are, the less symbols are able to convey meaning (visual expressiveness). This is because the human discrimination ability is limited (the span of absolute judgment that people can manage is of six categories). Increasing the number of conventions above this limit reduces understanding. Thus, a small number of graphical conventions will convey more meaning. The SEAM notation includes a rather manageable number of graphical symbols or semantic constructs (Table I). The Behavior view uses 6 types of service systems and 5 extra graphical symbols resulting in a graphic complexity of 11 . The Goal-Belief view uses the stickman and other 5 graphical symbols (Table I) yielding a total of 6 symbols. The SAR view uses 2 types of service systems (business and human) and 7 graphical symbols (Table I) resulting in a graphical complexity of 9 . From our experience, the 6 different graphical symbols used for service systems are very useful during workshops. Defining other graphical symbols for enterprise, committee, department, application and software component, and related conventions varies across workshops and is useful (e.g. same graphical symbol for all companies, but different color for services provided by each).

\section{Cognitive Fit}

Definition: "Different visual dialects should be used for different tasks and audiences". Notations should use different dialects for communicating with experts vs. novices [7].

SEAM models are mostly co-created during workshops (representation medium / production method: Post-its, schemas, pen and paper, posters) with the active involvement of participants. We recommend associating pictures to service systems and using the real names of the companies, people, and applications for a better mapping to reality. This helps 
participants understand models better. Simplified models can be created after the workshop for expert and novice audiences in computer-based drawing tools such as SeamCAD.

Furthermore, modelers use terms that are understood by the actors in the specific hierarchical level. At the business level, SEAM uses the business dialect for business people (e.g., organization, service, process, department) and the IT dialect (e.g., servers, infrastructure, computers, NAS, database, etc.) for IT people. This can be done in the same diagram (showing the IT department supporting the business and the business supporting the end client) or in separate models.

\section{J. SEAM Evaluation Summary}

To conclude our analysis, we provide a summary report [7]. We next formulate recommendations for improving the SEAM notation and discuss key concerns about PoNT.

\section{1) SEAM Evaluation with PoNT}

The SEAM notation performed very well for three of the nine principles:

Complexity management. Functional (from service / process as a whole to composite) and organizational (from service system as a whole to composite) refinement is used to manage complexity, showing hierarchies. In addition, colorcoding and grouping (systems' boundaries) help split one complex diagram into several simpler ones.

Dual coding. All SEAM graphical symbols are accompanied by short labels or descriptive textual expressions.

Cognitive fit. SEAM models are created for specific target audiences with groups of people sharing a common understanding of graphical symbols and semantic constructs. In the model hierarchy, business people would be more interested in the business aspects (top), whereas IT people would be interested in the IT configuration of components (bottom).

We identified improvements for six of the nine principles:

Semiotic clarity. Note the issue of level of semantic construct; 1:1 relationship between semantic constructs and graphical symbols for behavioral elements and 6:1 for service system. Same lines are used for different types of relations.

Perceptual discriminability. Consider texture and size for discriminating between symbols: background color for wholes vs. composites, size for large vs. small entities. This would be interesting for modeling power and influence relationships.

Semantic transparency. Improve icons to better suggest their meaning (e.g., goals, beliefs, IT, edges, values, features). Use metaphors for graphical symbols. Use graphical (not textual) distinction between wholes and composites (black for wholes and white for composites). Produce consistent icon types placed at the top right of each service system.

Cognitive integration. Include perceptual integration mechanisms, for instance explicit navigation through standardized storytelling showing the story with a short video and summarizing how the model was developed, not just the final version. Read diagrams from top to bottom or left to right.
Visual expressiveness. The SEAM notation includes the following visual variables: orientation (unique orientation for service systems, services and processes and any orientation for relations), value (labels for text), color (any), shape (boxes, oriented rectangles, diamonds, rectangles, circles, arrows, etc.). SEAM does not use or uses in an implicit manner the other variables: position (implicit wholes at the top and composites at the bottom), size (ad-hoc defined meaning) and texture (could be used to distinguish between wholes and composites).

Graphic economy. Use fewer constructs for Behavior and SAR models (currently 11 and 9). Remove the storage and component and consider them as IT systems. People appreciate them in workshops, as they were very well understood.

Overall, we can improve the SEAM notation using mainly semantic transparency, then visual expressiveness, semiotic clarity and graphic economy. We plan to involve end users to compare the SEAM notation with $i^{*}$, UML, BPMN, etc.

\section{2) Advantages and Limitations of PoNT}

PoNT focuses on the physical (perceptual) properties of notations, rather than their logical (semantic) properties. The nine principles were developed using a best-evidence synthesis approach and synthesized from theory (semiotic clarity) and empirical evidence (all other principles) about cognitive effectiveness of visual representations from a wide range of fields [7]. They were specifically designed for designing SE and RE notations and are based on general principles of visual perception and cognition applicable to any visual notations regardless of domain. Visual notations that (largely) satisfy the nine principles are more cognitively effective than those that do not. Thus, PoNT can be used to provide recommendations on visual notations and compare different visual notations.

We identified that PoNT makes no distinction between various contexts (business and IT models). It also focuses on the final models / diagrams but not on the process of creating them. PoNT does not consider human perceptions of reality (models are based on perceptions) or workshop resource limitations (time, tools, etc.). In our analysis, we noted that PoNT principles overlap: attempting to apply one principle often leads to the consideration of others. We observe that it is difficult to evaluate a hierarchical systemic notation with semiotic clarity. All of these concerns are representative of SEAM and cannot be addressed with PoNT.

\section{RELATED WORK}

In this section we argue on the merits of PoNT, as a scientific approach for evaluating, comparing, improving and designing visual notations. PoNT was successfully used to evaluate and improve several leading RE and SE notations: ArchiMate - international standard language for EA modeling, related to SEAM Behavior [7], UML - international standard language for modeling software systems, related to SEAM Behavior [9], i* - one of the most widely used goal modeling languages and leading requirements engineering / goal-oriented notations, related to SEAM Goal-Belief [8], BPMN - business process modeling international standard, related to SEAM Behavior [2], Use Case Maps - one of the most important user requirements notations with a long tradition in the field, related 
to SEAM Behavior [1], Business Decision Modeling - decision modeling notation developed by IBM aimed for business uses, related to SEAM Behavior and SAR [13].

The $i^{*}$ notation is designed to communicate with business stakeholders [8]. In $i^{*}$ designers model agent properties such as goals, beliefs, abilities, commitments, and strategic relationships. i* modeling results in high-level business models, without going into details about the processes and technologies. In [8], using the PoNT principles, the authors perform a systematic analysis of the visual syntax of $i^{*}$ identifying some serious flaws such as: zero visual distance for textual differentiation; too similar shapes for actor, agent, role and position and limited hierarchical structuring. The authors provide some recommendations for improvement such as: multiple levels of diagrammatic decomposition; use icons that suggest meaning, etc. SEAM Goal-Belief and i* models share numerous similarities, such as the type of graphical symbols they use, similar representations, etc.

BPMN uses flowcharting as a method for creating diagrammatic representations. Its notation relies on common sense, intuition and emulation of common practices, rather than on a rigorous scientific approach. Using PoNT the authors of [2] prove suboptimal (with respect to recommendation provided by the nine principles) language notation design decisions that may impede effective model-mediated communication between stakeholders proposing more semantically transparent graphical symbols, modularization with link events, etc. This was a first step towards making BPMN 2.0's visual notation more cognitively effective [2].

UCM enables the description of functional requirements as causal scenarios. Using the 9 PoNT principles Genon et al. [1] reveal some major issues with the current notation, such as no diagram level numbering, signposting or navigation maps, large graphic complexity (28 symbols, good only for experts), etc. The authors mention areas of improvement, such as reduce the symbol deficit (currently at $42 \%$ ), use size color and texture for perceptual discriminability, use conventional shapes for increasing the semantic transparency of 7 symbols, etc.

The Business Decision Modeling (BDM) tool from IBM supports the formalization of business processes in terms of decisions and rules that make up decisions. The main semantic elements are: decisions, business rules, operators, etc. In [13] the authors use the first 7 PoNT principles to evaluate BDM.

The SEAM notation comply with complexity management, graphic economy, cognitive fit better than BPMN, i* or BDM according to our experience. Future experiments will test this. Some issues are present for all notations, such as visual expressiveness, transparency and discriminability.

\section{CONCLUSIONS AND FUTURE WORK}

The Physics of Notations Theory helps evaluate, compare, improve and design visual notations in a wide variety of fields. Inspired by a number of research publications that use PoNT to evaluate and improve several leading RE and SE notations, we have discussed the main implications of evaluating a systemic method, the Systemic Enterprise Architecture Methodology
(SEAM) with all of the nine PoNT principles, and we have proposed a few suggestions to improve the method's visual notation. Some principles apply more extensively to the SEAM nature whereas others provide only limited insights being partially applicable. Given its systemic nature, the SEAM notation is well fit to represent business and IT models that are cognitively effective in communicating analysis and design decisions. We have presented suggestions for improvements for six out of nine PoNT principles. We promote the evaluation with PoNT of other SE and RE methods. PoNT can complement other methods, such as heuristic evaluation and usability tests. Next, we plan to perform notation-evaluation experiments with users, study the evolution of the SEAM notation throughout a project lifecycle and extend PoNT with systemic principles. In this paper, the different views of SEAM (Behavior, Goal-Belief and SAR) were considered independently. In the future, we will evaluate the integration between these models, thus studying the relationship between motivation, service-process and component-feature-value.

\section{REFERENCES}

[1] Genon, N., Amyot, D. and Heymans, P. Analysing the cognitive effectiveness of the UCM visual notation. In Proceedings of the 6th international conference on System analysis and modeling: about models, Frank Alexander Kraemer and Peter Herrmann (Eds.). SpringerVerlag, Berlin, Heidelberg, 221-240. 2010.

[2] Genon, N., Heymans, P. and Amyot, D. Analysing the Cognitive Effectiveness of the BPMN 2.0 Visual Notation. In: Malloy, B., Staab, S., van den Brand, M. (eds.) SLE 2010. LNCS, vol. 6563, pp. 377-396. Springer, Heidelberg. 2011.

[3] Green, T. R. G., Blandford, A. E., Church, L., Roast, C. R. and Clarke, S. Cognitive Dimensions: Achievements, New Directions and Open Questions. J. Visual Languages and Computing, pp. 328-365, 2006.

[4] ITU T X.902 / ISO/IEC 10746-2 Information Technology - Open Distributed Processing - Reference Model - Foundations. 2010.

[5] Krogstie, J., Sindre, G. and Jørgensen, H. Process models representing knowledge for action: a revised quality framework, European Journal of Information Systems 15 (1), 91-102. 2006.

[6] Mendling, J., Reijers, H. A. and van der Aalst, W. M. P. Seven Process Modeling Guidelines (7PMG). Information and Software Technology Journal. 52 (2), 127-136. 2010.

[7] Moody, D. The Physics of Notations: A Scientific Approach to Designing Visual Notations for Requirements Engineering. Requirements Engineering Conference, Atlanta, Georgia, U.S. 2009.

[8] Moody, D. L., Heymans, P. and Matulevicius, R. Visual Syntax Does Matter: Improving the Cognitive Effectiveness of the $i^{*}$ Visual Notation. Requirements Engineering 15(2), 141-175. 2010.

[9] Moody, D. L. and van Hillegersberg, J. Evaluating the Visual Syntax of UML: An analysis of the Cognitive Effectiveness of the UML Family Of Diagrams. Conference on Software Language Engineering. 2008.

[10] Porter, M. E. Competitive Strategy: Techniques for Analyzing Industries and Competitors. New York: The Free Press. 1980.

[11] Regev, G. and Wegmann, A. Where do Goals come from: the Underlying Principles of Goal-Oriented Requirements Engineering. Requirements Engineering Conference, Paris, France. 2005.

[12] Regev, G., Bajić-Bizumić, B., Golnam, A., Popescu, G., Tapandjieva, G., Saxena, A. B. and Wegmann, A. A Philosophical Foundation for Business and IT Alignment in Enterprise Architecture with the Example of SEAM. BMSD. 2013.

[13] Thomas, J. C., Diament, J., Martino, J. and Bellamy, R. K. E. Using the "Physics" of Notations to Analyze a Visual Representation of Business Decision Modeling. Visual Languages and Computing. 2012.

[14] http://www.tradeyourmind.com/ - Web version of SEAM. 2014. 